\section{Packed full of natural ingredients}

Curaprox is passionate about offering environmentally friendly oral healthcare solutions that are gentle on teeth and gums, but tough on plaque.

With six exciting flavours to choose from, 'Be you' toothpastes embody this ethos with a formula that does not contain animal products, nor any SLS, triclosan, bleaching agents or plastic microbeads.

Dental professionals can recommend 'Be you' toothpastes knowing that they are packed full of natural ingredients, including extracts of Echinacea, bitter orange, devil's claw and Indian pennywort, which are designed to nourish the oral tissues.

Glucose oxidase - the natural enzyme contained in 'Be you' toothpastes - also ensures a gentle whitening effect for a brighter, healthier smile.

Call now to order.

For more information call 01480 862084, email info@curaprox. co.uk or visit www.curaprox.co.uk.

\section{For elevated restorations}

LM Dark Diamond coated instruments have a non-stick coating for the easy placement and contouring of composite resins.

LM is always looking for ways to push boundaries and make life easier - with Dark Diamond the diamond-like coating means that efficiency as well as precision will be optimised.

Other features:

- A hard, smooth surface that is easy to clean and is scratch-resistant

- Maximum contrast against the filling and minimal reflections

- An ErgoSense handle - LM has always been a pioneer in ergonomics.

LM Dark Diamond instruments are available from J\&S Davis, the distributor of high-quality dental products. Contact the team direct or your preferred dental dealer for more information.

Find out more today by visiting www.js-davis.co.uk, calling 01438 747344 or emailing jsdsales@js-davis.co.uk.
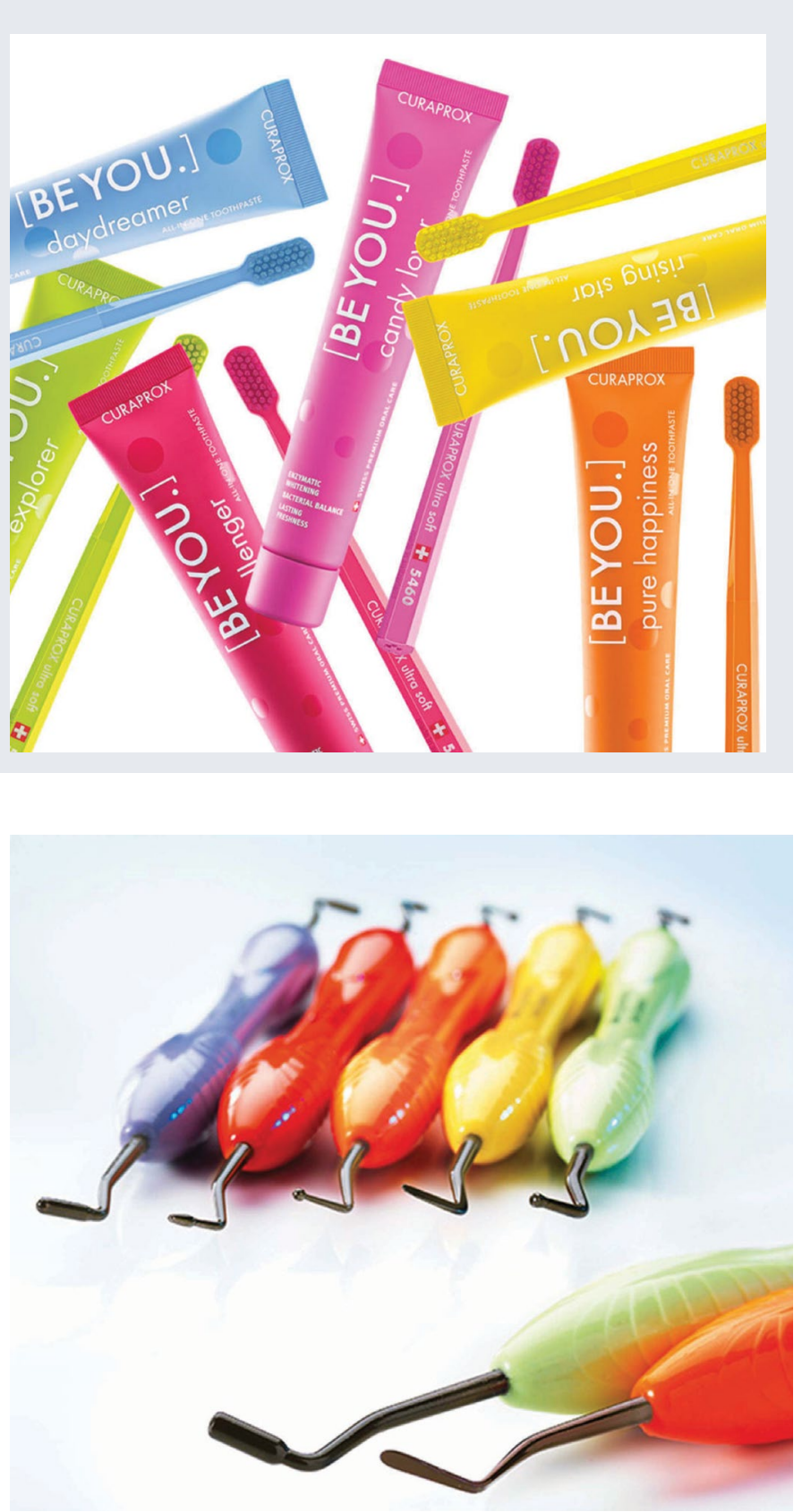

\section{Comfort and efficiency}

If you're making a recommendation of root canal therapy, the only thing a patient might want to know is, 'Will it hurt?'

COLTENE supplies all the tools and materials for comfortable, efficient and successful endodontics.

The range includes HyFlex EDM files, for all cases, whether straightforward or complex situations. Available in different sizes, each one offers up to $700 \%$ more fracture resistance than other similar solutions. For retreatments, the REMOVER file will mechanically clean away obturation material, no solvent required.

When you choose COLTENE, you can reassure your patients that there is no need to worry as it will be high-quality, high-value treatment, performed with their comfort in mind.

For more on COLTENE, visit www.coltene.com, email info.uk@ coltene.com or call 08002545115.

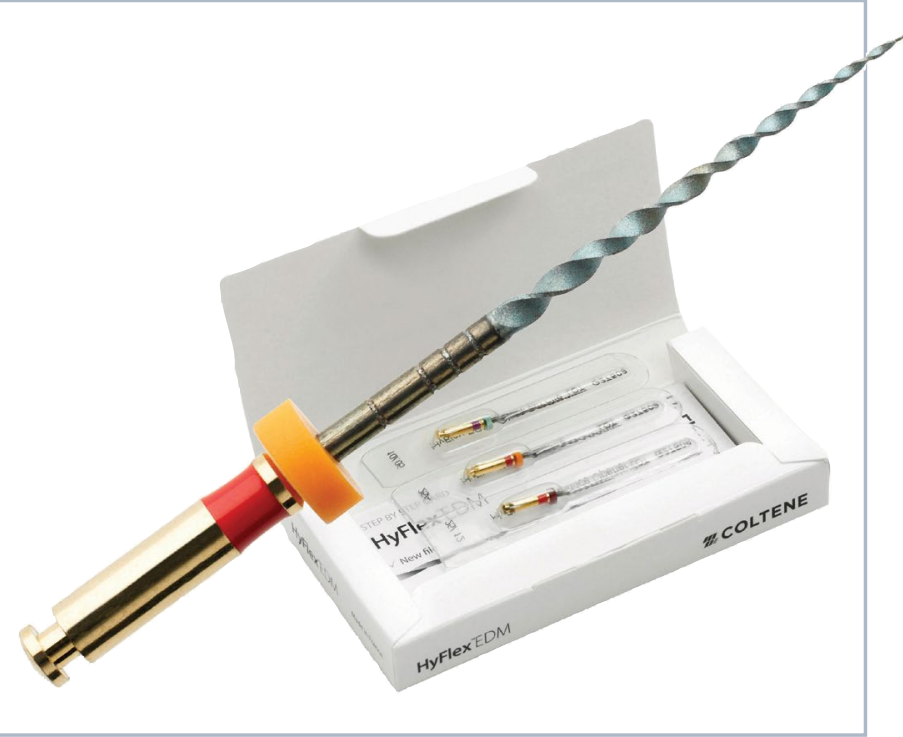

